\title{
Social Sustainability Achievement through Mobile GIS Applications Development and Audit
}

\author{
Cosmin TOMOZEI ${ }^{1}$, Cristian AMANCEI $^{2}$ \\ ${ }^{1}$ Vasile Alecsandri University of Bacău, Romania \\ ${ }^{2}$ Bucharest University of Economic Studies, Romania \\ cosmin.tomozei@ub.ro,cristian.amancei@ie.ase.ro
}

This paper aim is to determine a reliable way for the mobile health applications engineering, reengineering and audit, for the achievement of social sustainability. This research implied the constructive analysis of health applications of several types, by means of scientific databases, in which GIS and mobile applications for health were presented. Secondly, the use of mobile devices and GIS applications for senior citizens have been taken into account so as to create efficient ways of communication and processing of medical and spatial data. These types of applications should provide help and support for people in need and assist the decision makers, both patients and health specialists for the assurance of the quality of life as well as the seniors' autonomy. A detailed presentation of the results of this research was included in a lecture at the Hanzehogeschool University of Applied Sciences of Groningen, Netherlands, within the Erasmus+ European teaching program in May 2015.

Keywords: Mobile Health, GIS, Sensors, Data Analysis, Audit

1 Introduction

As it represents a key-element of development, social sustainability includes concepts such as livability, health and community development, in this context the research about the healthy ageing phenomena and the friendliness of communities becomes an imperative. This research brings into play the development of mobile geographic information systems - GIS applications in the evolution of social requirements regarding the increase in the quality of life and autonomy of certain communities.

The paper tries to offer a reliable answer to the questions whether the exploitation of mobile applications is beneficial for the senior citizens and if so what are the ways in which these applications should be developed so as to assure the best user experience. The concepts of citizen oriented applications [12] and usability are to be defined and described in a particular manner, in order to correspond to the social sustainability requests of specific communities.

Another essential side of this research is to conclude if the process of software reengineering is appropriate for the radical transformation of legacy desktop software to mobile applications and how to add the mapping and GIS features, which are necessary for the fulfilment of novel social and economic objectives.

The fact that the target group is the citizens of advanced age involves drawing some special requirements regarding the structure of user interfaces, user session duration and number of steps needed to meet immediate objectives. Making custom recommendations [9] and establish daily dosages to optimize of drug treatments, as well as notifying users about activities at certain times represent just a few of the functionalities these types of applications needed to increase the independence of the seniors.

Some of the best-known aspects of sustainability achievement concerning the modern society are represented by age friendly communities and by the easiness of access to the resources by the senior citizens. In papers such as [1] the age friendliness of cities is analyzed, especially by taking into account the allocation of health resources. The knowledge-based society has as a main objective to offer the people in need the appropriate health resources and services in a suitable time, furthermore to support the autonomy and possibility of elderly to live in 
a secure, healthy and friendly environment, which presumes that they should be able to work with the new information and communication technologies so as to provide valuable information to the medical staff and to their families.

In [1] the friendliness of city is analyzed by means of two main areas, such as the needs of the elderly and the capacity of the community to cover those needs. In order to create a clear view of the population lifestyle and health, indicators have been defined which measured the diets, movement and habits. In our case, specific indicators should be determined as well by means of mobile GIS applications specially build for this category of citizens. The use of mobile applications should be available for the grand majority of seniors in certain geographic areas. In some cases, either because of the lack of resources or because of the lack of digital education problems may appear. Consequently, some percentage of the senior citizens will not be included in the statistics. The exposure of the seniors to natural and anthropic risks may affect also their life quality and autonomy.

In order to accomplish the social goals of healthy aging and to increase the life expectancy, it is very important that the data which result from the interaction of the senior citizens with the technological devices to be subjected to analytical procedures as well as the extraction of the most significant information, by the specialists, though data modelling and estimation procedures.

Such procedures are identified in [2], in which exposure to environmental risk factors is assessed. The thematic maps are based on the geocoding process and on and on the utilization of GPS devices, sensors and databases. Valuable data, obtained by smart devices and sensors are processed and transmitted to the health specialists. Based on that data, the evolution of cities designed is to be predicted and future development of geographic areas is to be estimated as well. As 75 percent of the population in Europe live in urban areas and the majority of them in cities between 150,000 and 500,000 habitants, their environmental and health risk exposure is very consistent and certain measure should be continuously taken, based on statistics analysis of impact on population. However, this also means that the grand majority of Europe's population has the possibility of being connected to the services provided to mobile devices by the communities and by the health organizations.

On the one hand, the geographic information systems offer a reliable support for data representation and analysis, by integrating specific procedures, such as spatial analysis and regression, time series, charts and geocoordinates, which are very helpful in the development of health graphical representations on thematic maps. As tablets and intelligent devices are greatly used in healthcare, as mentioned in [3], for general practitioners and patients, aiming to reduce the incidence of strokes and monitor the cardiovascular diseases, additional functionalities may be taken into account, for the spatial analysis of diseases and for the creation of estimation models. Smart devices are appropriate for mapping software and by means of touchscreens, gestures and sensors a set of new indicators is in position of being developed.

On the other hand, statistic and econometric models for descriptive analysis, estimation of causal relations and prediction should be developed based on the data gathered through mobile devices and application from the senior citizens. Based on the models which are to be obtained, recommendations must be given to the community members from certain geographic areas by the specialists from different areas of studies and different domains of activity such as health, research or public administration.

A novel approach of healthy aging application development consists of the events which are implemented by software functionalities, which should be used for elderly people interaction with the devices by tap, double tap, tap-and-hold, pan, slide or flip which are very usable and comfortable in the user interaction. These events or gestures combined with geolocation facilities and data obtained through sensors becomes more and more 
beneficial in mHealth [4] applications development. The mHealth area of research presumes the integration of the works of many specialists belonging to many areas of study, such as biomedical engineering, computer science, medicine and statistics. The need of common work and homogenous approaches in healthy ageing software development entails the need of high quality research.

It is stated that the mHealth phenomena got significant attention in the last years, because of the devices that support the health objectives and transformed the health services and their accessibility for the patients. Complex communication functionalities have been integrated, by connecting medical devices via $\mathrm{Wi}-\mathrm{Fi}$ and Bluetooth for the transmission of data regarding the physical condition of the patients, such as blood pressure, glycaemia, body temperature, heart rate but further analyses are to be accomplished for more elaborated examinations and calculations.

Examples of smart devices with health capabilities and sensors are the physical monitors developed by Polar [14], which include GPS and training features as well as 24/7 monitoring of daily activity and heart rate, the Fitbit [15] heart rate and activity tracker and iHealth [16] wireless glucometers blood pressure monitors and fitness devices. The data provided by these devices trough their sensors in conjunction with mobile devices, such as smartphones and tablets may further collected and analyzed for the adoption of healthy aging decisions.

\section{Citizen assistance, healthcare and quality of life}

The assurance of a good public health system represents a continuous aim of researchers and practitioners, so as to draw-up the characteristics and the necessity of well-being for the elderly people. Measures have been taken [5] for the building of healthy living environments by combining medical treatments with movement and physical exercises. Furthermore, studies and policies about the "aging-in-place" have been created in order to contribute to the people's autonomy in life. As people advance in age, they are about to spend more time in their homes and community environments. The health deterioration and the functional disabilities may become barriers in senior citizens daily lives subjecting them to difficult situations from which they should exit with the support of mobile applications and GIS.

Mobile applications and remote sensors e.g. smart wearable devices and pedometers support the development of healthy communities and age-friendly environments. Several factors such as walkability, air quality and green space as well as reduced levels of danger have been considered as vital for age friendly environments and the urban planners and designers should be considered as important elements for the planning and design work. In addition to the ergonomics and the secure environment which should be assured for the ageing communities, an important element consists of the assurance of lifelong health assistance system.

Personal health information should be in position of being modelled with the support of smart devices and customized for the personal health requirements, in order to assist [6] the seniors for the maintenance of life quality and health condition. Citizen oriented health assistance services are continuously lifelong provided and assure the possibility of managing their needs and treatments, with health practitioners advisory services approval.

Mobile health applications for the seniors should have a certain level of autonomy and mainly use the computing capabilities of the devices they are installed on. Only the sets of data which are essential for the analysis should be sent to processing servers, due to information privacy issues, servers overload and traffic costs.

Mobile health and the integration of sensors and wearable technologies offer to the elderly people new ways of connectivity and interaction, within a smart medical distributed system for uninterrupted healthcare assistance. Furthermore, clinical professionals utilize such assistance systems in order to support the elder patients' home care. 
These services are accessible via dedicated protocols and their functionalities are exposed to the mobile devices which the patients carry in their daily lives. An important issue is represented by the success in communication between the clinical specialists, the software development teams and the beneficiaries concerning the objectives, the personal health plan structures, the data formats and the data exchange between the actors by means of specialized services.

Another important issue is related to the data storage and the distribution between specialized servers and Cloud machines, for large files and objects such as medical imaging files, and personal mobile devices, with a direct effect on how data is queried and structured in the following stages for the specialized medical decision support activities. Automated reasoning procedures may be implemented and adapted for the creation of personalized recommendations based on each person activity history and health records. Service oriented architectures and service composition models for health management are being implemented and invoked by mobile health applications.

The mobile devices are enhanced with mapping engines and high performance sensors which are to be used in the development of mHealth GIS. Structuring and georeferencing the information provided by the patients becomes a must and allows the development of correlation analysis between certain geographic areas where communities are placed and any environmental or health risks which may occur.

\section{3 mHealth applications engineering for senior citizens}

A reliable taxonomy of mHealth applications is presented in [4] and offers a holistic image about the objectives, engineering, maintenance and reengineering of health applications. Organizations adopt more and more mHealth apps for the increasing of the patients' satisfaction regarding the medical and social services. These applications deeply contribute to the autonomy of the patients and support the achievement of high levels of the life quality.

In this section, we present a simple example for mobile application with use in healthy aging and especially with regards on the quality of life of the senior citizens.

For elderly people the consumption of water, salt, coffee, prescription medication, as well as the movement per day represent key elements for a healthy and autonomous life. These factors, identified as exogenous variable in a regression model, may straightforwardly be modeled by means of mobile devices and services, in a mobile-first cloud first strategy of going forward, according to [10].

Blood pressure is taken into account as an endogenous variable, modelled in terms of the identified exogenous variables, by means of classes in the mobile health applications. The classes and the mHealth services are instantiated on the mobile devices. The identified linear regression model will be useful in the estimation of the blood pressure based on the quantities of products which the patients had consumed and consequently inplace recommendations will be given to the patients.

A secondary phenomenon which assures the quality of life of senior citizens is represented by the movement per day variable. The movement per day a citizen does is measured by pedometers and activity trackers wearables and gets easily modeled by mobile devices via Bluetooth connections. The movement per day is depending on the weather and especially on the temperature, because it is advisable that the elderly people should avoid the outdoor activities on very cold or very hot weather. Another aspect regarding the values of the movement per day factor is determined by the geographical location of the citizen towns of residence. This leads to the adoption of the GIS modules and furthermore on the integration of map modelling procedure on mHealth application based on a process of software reengineering.

Time series and regression analysis are key elements in the analysis of health indicators and in the estimation of health data models. Limits should be set by medical specialists 
and those limits should not be exceeded by the patients in their daily life.

In (1), (2) and (3) the regression models for the blood pressure and movement per day are analytically defined and described as simultaneous equations models.

$$
\begin{array}{r}
\text { Blood_pressure } e_{k}=f(\text { salt,coffee, water,medication })_{k} \\
\text { Movement_per_day }_{k}=f(\text { temperature, geo_position })_{k} \\
B P_{k}=\operatorname{VarEx}_{k 1} \beta e t a_{k 1}+\sum_{j=1}^{N r E x} \operatorname{VarEx}_{k j} \beta e t a_{k j}+u_{k}
\end{array}
$$

where:

- $B P_{k}$ is the dependent, endogenous variable of ecuation $k$ within the model, identified as blood pressure;

- $\quad \beta e t a_{k 1}$ is the intercept of the simultaneous equations model;

- $\quad \operatorname{Beta}_{k, l}$ is the coefficient of the independente variabile Varex $_{\mathrm{k} l}$, of the model;

- $u_{k}$ is the error term.

The optimization of the model leads to personalized recommendation of quantities for each of the exogenous variables, e. g. salt, medication, water and coffee in order to support the positioning of the blood pressure in the normal limits. The models should be structurally consistent and avoid multicollinearity. The size of the model is determined by the number of exogenous variables and the number of data series. This analysis of multicollinearity checks whether there is correlation between the explanatory variables which lead to the inconsistence of the model.

An additional aspect, which is significant for the quality of life is represented by electronic prescriptions, which are directly sent on mobile devices, back ended in Cloud and with push notifications. The electronic prescription sent on mobile devices with notification will considerably decrease the time spent by the elderly people as well as the effort to get the necessary medication for the assurance of their quality of life.

\section{Mobile development of health GIS applications for senior citizens}

The mHealth GIS application prototype is being built by taking into account the premises of assuring the lifelong autonomy of the elderly. The application is being developed on the .NET Framework and includes the following functionalities:

- the geo position finder and emergency service notification system;

- the activity tracker - movement per day, which integrates data obtained through sensors and wearable technologies, such as Polar and Fitbit;

- the medication tracker and notification system, based on the prescriptions and the treatments given by the medical practitioners;

- the go to sleep recommendation notification functionality, within the daily activity of each senior citizen;

- the have some water recommendation, for the monitoring of the water quantity consumed by each person per day;

- the go to the family doctor reminder; the elderly should go to their family practitioners at certain intervals of time in order to renew their prescriptions;

- the coffee and salt monitor, especially in the case of hypertension;

- the environmental risk GIS analysis and risk graph [13] creation engine;

- the geospatial regression analysis engine, for the estimation of risk occurrence situations and weather forecast data processing. 


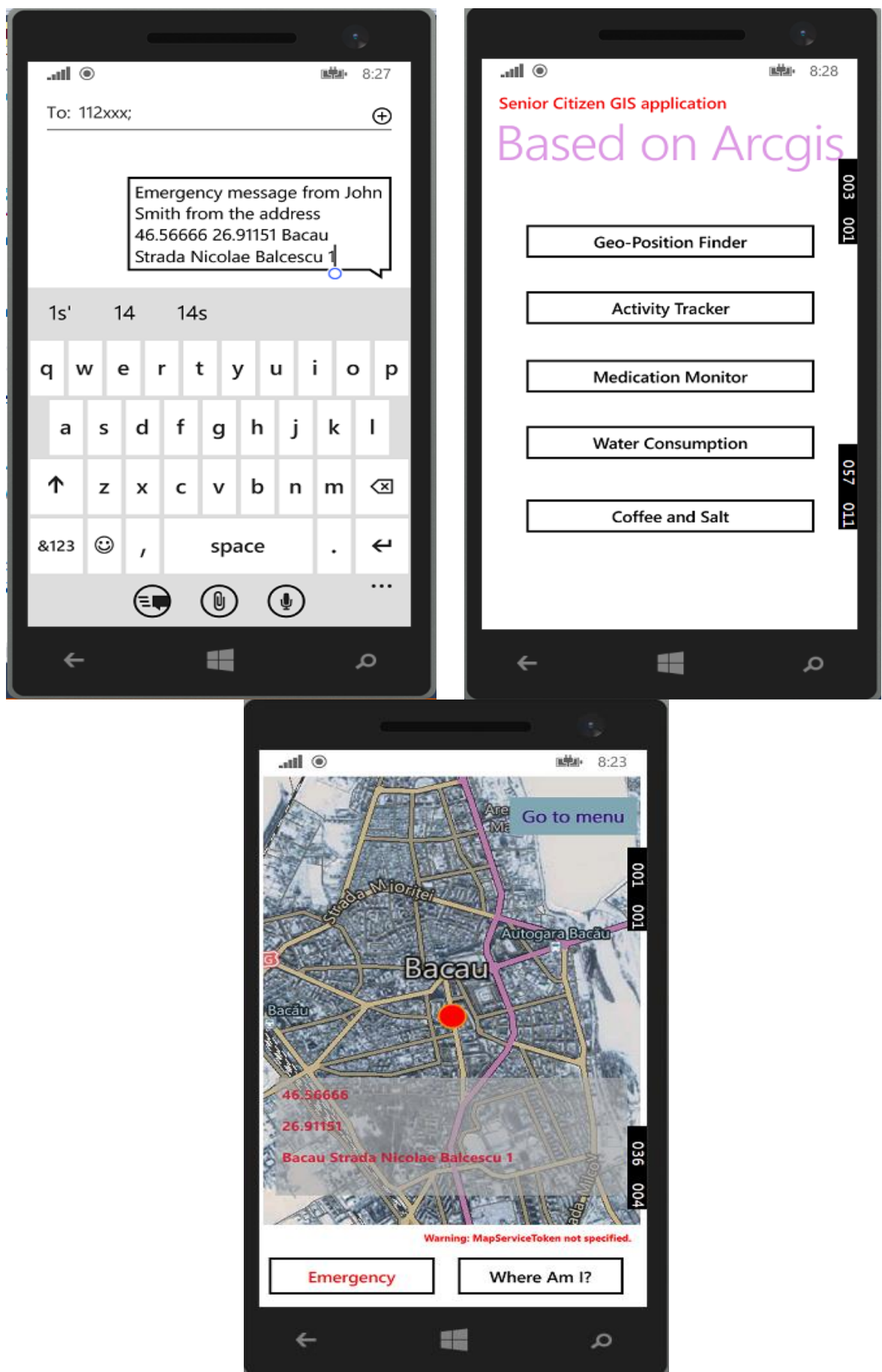

Fig. 1. Windows Phone Mobile application for senior citizens

These functionalities are implemented by classes which are written in the Visual C\# language on the .NET framework and involves the use of ArcGIS Runtime SDK for Windows Phone 8.1, available at [17]. The Microsoft mapping services are also used in conjunction with the services from ESRI.

The geo-position finder and emergency notification system is presented in Figure 1. In case of need, the elderly have the possibility to tap on the "Where am I?" button and to determine exactly, in seconds their geoposition and furthermore the geo coordinates are also available for being sent to the authorities.

The asynchronous method for the geolocation identifying process is presented below: 


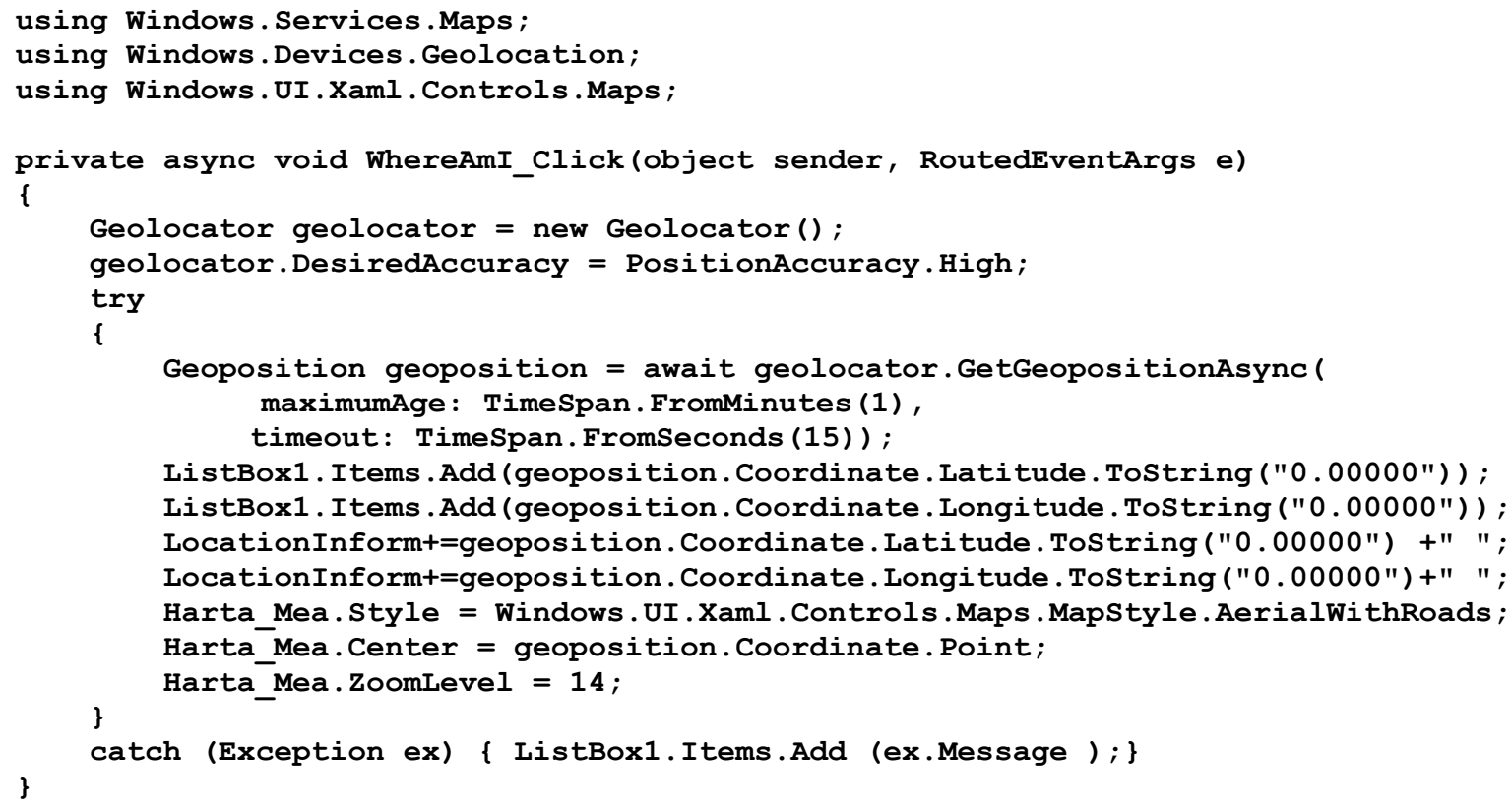

Consequently, by pressing the emergency button, an SMS message will be composed automatically, which contains the data of the persons in need, such as the name of the persons, the geographic coordinates where they are placed at, the address (name of the city, name of the street and street number). The message is very easy and intuitive to be sent to the authorities. This process is aimed to be more efficient in comparison to the Call 112 approach and valuable time may be saved. Another important aspect is based on the accuracy of the data which is sent, by being automatically collected and processed, without human intervention and without human error. The SMS notification asynchronous method for emergency is presented below:

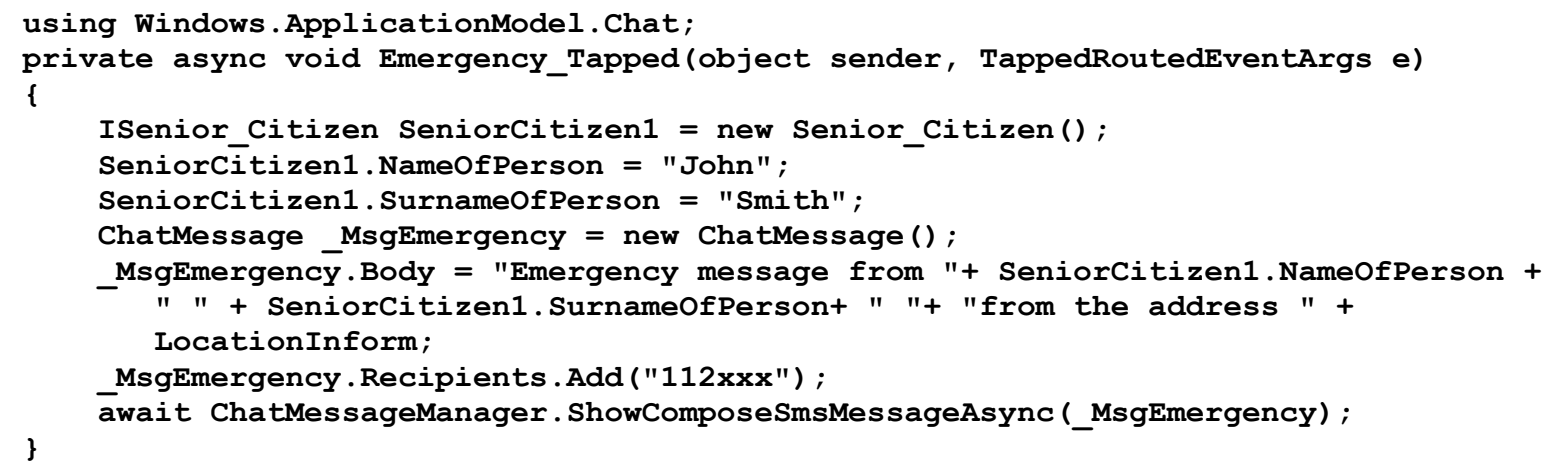

The implementation of these functionalities presumes that the .NET Framework and the Visual C\#.NET programming language is used. Both methods from figure 2 and figure 3 are handling the tap and click events, as on screen gestures on Windows Phone 8.1 mobile devices. Both methods are asynchronous, which means that they do not block the execution of the application, which continues with the other processes that do not rely on the same resources. Objects such as
Geolocator and Geopositions are being instantiated and implemented in the application for the efficient access of the map control functionalities.

\section{5. mHealth application audit approach}

In this section, we present the audit approach proposed for our mobile application architecture proposal.

The audit starts with a security risk analysis main focused on analysing vulnerabilities and 
threats to the mHealth information resources in order to decide what controls should be implemented in the future development phases. Due to the complex interactions among the components of the information system, a single vulnerability may have multiple propagation paths, leading to different security risks for our application [11].

Based on a self-assessment of the patient information manipulated by the application, we have developed a list of main risk factors and proposed mitigation controls presented in Table 1.

Table 1. mHealth main risk factors and proposed mitigating controls

\begin{tabular}{|c|c|c|}
\hline Category & Risk factor & Proposed mitigating control \\
\hline \multirow[t]{3}{*}{$\begin{array}{l}\text { Application } \\
\text { security }\end{array}$} & $\begin{array}{l}\text { Application access } \\
\text { control }\end{array}$ & $\begin{array}{l}\text { Access to the analysis results will be permitted } \\
\text { through accounts validated by the application } \\
\text { administrator. } \\
\text { Access to personal data will be permitted only } \\
\text { through authentication with digital certificates. }\end{array}$ \\
\hline & $\begin{array}{l}\text { Application processing } \\
\text { history }\end{array}$ & $\begin{array}{l}\text { Logs will be maintained for the processing } \\
\text { history of the application at a high detail level. }\end{array}$ \\
\hline & $\begin{array}{l}\text { Capability of fault } \\
\text { tolerance }\end{array}$ & $\begin{array}{l}\text { All the client requests will be parsed and the } \\
\text { application will respond only to a predefined } \\
\text { set of utilization cases, for all the other requests } \\
\text { general messages will be presented. }\end{array}$ \\
\hline \multirow[t]{3}{*}{ Data security } & Data confidentiality & $\begin{array}{l}\text { The access to the database will be restricted } \\
\text { only to trusted connections that are enforced by } \\
\text { digital certificates, due to the sensitivity of the } \\
\text { data stored. }\end{array}$ \\
\hline & Data integrity & $\begin{array}{l}\text { Data upload will be permitted only for the } \\
\text { administrative users that remain responsible to } \\
\text { ensure the integrity of the data. The client } \\
\text { application interface will permit only } \\
\text { operations that do not involve data upload. }\end{array}$ \\
\hline & $\begin{array}{l}\text { Data transmission } \\
\text { controls }\end{array}$ & $\begin{array}{l}\text { The data input gathered from the client } \\
\text { interface will be checked for reasonableness. }\end{array}$ \\
\hline \multirow[t]{2}{*}{$\begin{array}{l}\text { Communications } \\
\text { and operation } \\
\text { security }\end{array}$} & $\begin{array}{l}\text { Communication } \\
\text { integrity }\end{array}$ & $\begin{array}{l}\text { Communication integrity will be protected by } \\
\text { encryption. The level of encryption will be } \\
\text { generated by the sensitivity of the } \\
\text { communicated information. }\end{array}$ \\
\hline & $\begin{array}{l}\text { Protection of log } \\
\text { information }\end{array}$ & $\begin{array}{l}\text { The application and system log files will be } \\
\text { stored on a dedicated machine with a different } \\
\text { permission set than on the production } \\
\text { environment. }\end{array}$ \\
\hline \multirow[t]{2}{*}{$\begin{array}{l}\text { Application } \\
\text { processing }\end{array}$} & $\begin{array}{l}\text { Audit trails and } \\
\text { overrides }\end{array}$ & $\begin{array}{l}\text { Automated tracking of changes made to data, to } \\
\text { a specific user. Also, the override of normal } \\
\text { application processes will be logged separately } \\
\text { by the system. }\end{array}$ \\
\hline & Interface balancing & $\begin{array}{l}\text { Automated checking of data received from } \\
\text { client system into the database for } \\
\text { completeness, through balance reconciliation. }\end{array}$ \\
\hline
\end{tabular}

After application development is finished we will perform detailed testing of the main risk factors identified in order to check if the proposed mitigating controls are appropriate 
and sufficient for our application.

\section{Conclusions}

Mobile devices are accessible, powerful and useful for data processing and transmission without a substantial effort by the elderly people, by means of specific mobile software applications. Mobile health applications become more and more present in people's lives and provide useful information to the health specialists.

Our paper intention is to reflect a novel utilization of this types of devices for the support of the senior citizens autonomy and quality of life, through software engineering and reengineering strategies. Health services and service oriented architecture play a decisive role in this context and assure the communication between mobile device applications and specialized servers.

The engineering of the mobile apps presumed the definition of models in which endogenous variables were explained in terms of exogenous, independent variables in the regression analysis, based on the data collected by mobile devices and wearable servers connected via Bluetooth and Wi-Fi.

The knowledge-based society has as a main objective to offer the people in need the appropriate health resources and services in a suitable time and therefore to provide the health facilities, medication and accurate information, so as to lead to an increase of personal autonomy and the possibility of living in a secure, healthy and friendly environment. Mobile health apps maintain this desire achievable and accessible to many people.

\section{Acknowledgement}

A shorter version of this paper was presented at the $14^{\text {th }}$ International Conference on Informatics in Economy (IE 2015), May 1-3, 2015.

\section{References}

[1] J. Ruza, J. I. Kim, I. Leung, Ca. Kam and S. Y. Man Ng, "Sustainable, age-friendly cities: An evaluation framework and case study application on Palo Alto,
California", Sustainable Cities and Society, vol. 14, pp. 390 - 396, 2014.

[2] D. Fecht, L. Beale and D. Briggs, "A GISbased urban simulation model for environmental health analysis", Environmental Modelling \& Software, vol. 58, pp. 1-11, 2014.

[3] M. Radzuweit and U. Lechner, "Introducing tablet computers into medical practice: design of mobile apps for consultation services", Health and Technology, vol. 4, No. 1, pp 31-41, 2014.

[4] P. Olla and C. Shimskey, "mHealth taxonomy: a literature survey of mobile health applications", Health and Technology, vol. 4, No. 1, pp 31-41, 2015.

[5] E. J. Burton, L. Mitchell and C. B. Stride, "Good places for ageing in place: development of objective built environment measures for investigating links with older people's wellbeing", $B M C$ Public Health, Vol. 11, 2011, Available: http://www.biomedcentral.com/14712458/11/839.

[6] S. C. Christopoulou, "A smart citizen healthcare assistant framework", Health and Technology, vol. 3, No. 3, pp 249-265.

[7] J. Kerr, S. Duncan, J. Schipperjin, "Using Global Positioning Systems in Health Research A Practical Approach to Data Collection and Processing", American Journal of Preventive Medicine, vol. 41, No. 5, pp. 532-540, 2011.

[8] A. K. Lyseen, C. Nøhr, E. M. Sørensen, O. Gudes, E. M. Geraghty, N. T. Shaw, C. Bivona-Tellez, "A Review and Framework for Categorizing Current Research and Development in Health Related Geographical Information Systems (GIS) Studies", Yearbook of Medical Informatics, Available: http://www.ncbi.nlm.nih.gov/pubmed/25 123730

[9] N. Vercruyssen, C. Tomozei, I. Furdu, S. Varlan, C. Amancei, "Collaborative Recommender System Development with Ubiquitous Computing Capability for Risk 
Awareness", Studies in Informatics and Control, vol. 24, No. 1, pp. 91-100, 2015.

[10] Satya Nadella: Mobile First, Cloud First

Press Briefing, Available: http://news.microsoft.com/2014/03/27/sat ya-nadella-mobile-first-cloud-first-pressbriefing/

[11] N. Feng, H. J. Wang, L. Li, "A security risk analysis model for information systems: Causal relationships of risk factors and vulnerability propagation analysis", Information Sciences Journal 256/2014, pp. 57-73

[12] I. Ivan, B. Vintilă, C, Ciurea, M. Doinea, "The Modern Development Cycle of Citizen Oriented Applications",
Informatica Economică Journal, Vol. 18, No. 3, pp. 263-270, 2009.

[13] M. Talmaciu, V. Lepin Algorithms for the Recognition of Net-free Graphs and for Computing Maximum Cardinality Matchings in Claw-free Graphs, Studies in Informatics and Control, Vol. 23, No. 3, pp. 183-188, 2014.

[14] Polar, Available: http://www.polar.com/en/products
[15] Fitbit, Available: http://www.fitbit.com/home
[16] iHealthlabs, Available: http://www.ihealthlabs.com/glucometer/
[17] ArcGIS for Developers, Available: ESRI, https://developers.arcgis.com/en/

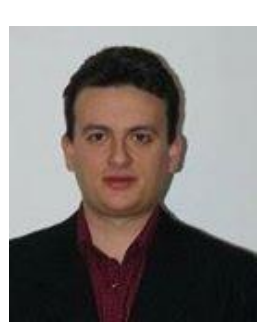

Cosmin TOMOZEI is assistant professor at Vasile Alecsandri University of Bacău, Romania, at the Department of Mathematics, Informatics and Education Sciences. He has obtained the PhD Economic Informatics, at the Doctoral School of the Bucharest University of Economic Studies, Faculty of Economic Cybernetics, Statistics and Informatics, Department of Economic Informatics and Cybernetics in 2012. His research area is centred on the field of software engineering and reengineering, with applications in Government, Education and Health. Between the years 2006 and 2008 he attended the Master in Economic Informatics courses, the Databases and Business Support study programme, organized by the Bucharest University of Economic Studies. In 2006 he acquired the Bachelor degree in Economic Informatics, at the Faculty of Economic Cybernetics, Statistics and Informatics, from the Bucharest University of Economic Studies.

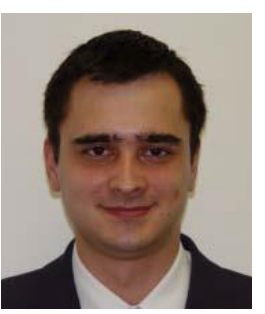

Cristian AMANCEI is assistant professor at Bucharest University of Economic Studies, Faculty of Economic Cybernetics, Statistics and Informatics. He has obtained the PhD Economic Informatics, at the Doctoral School of the Bucharest University of Economic Studies, Faculty of Economic Cybernetics, Statistics and Informatics, Department of Economic Informatics and Cybernetics in 2011. He holds a Master in Science - Computerized Project Management from Academy of Economic Studies, Bucharest. He is Certified Information Systems Auditor (CISA), Certified Information Systems Security Professional (CISSP). He graduated in Economic Informatics at Faculty of Economic Cybernetics, Statistics and Informatics in 2006. His main research areas are: information system audit, metrics in information systems, IT controls and IT risks. 